$50 \%$, they speculated that it might be related to an effect on $\mathrm{NF \kappa B}$, and give circumstantial evidence supporting this view.

\title{
Acquired resistance of human T cells to sulfasalazine
}

\section{E Furst}

\section{Have we found a reason why patients become "resistant" to DMARDs?}

C inicians have the impression that patients may respond to a given drug (DMARD) for some period of time, then become "resistant", a situation in which these drugs seem to lose their clinical effect. In this month's Ann Rheum Dis, Joost van der Heijden and colleagues describe acquired resistance to sulfasalazine (SSZ) through an effect on a drug efflux pump. They then go on to describe the kinetics of this mechanism and test the effect of this change on other DMARDs.

In general, the efficacy, or lack thereof, of DMARDs may be due to a very large number of factors. The mechanism of action of drugs in a given disease, of course, plays a major part in its efficacy and is dependent upon the pathophysiology and pathogenesis of the disease. In addition, the pharmacokinetics of the drug have a role in drug effects, which includes absorption and delivery of the drug to its site of action and its removal from the site by its distribution/transportation, metabolism, and/or excretion. In addition, the patient's genetic background, comorbid diseases, and concomitant drugs may have effects on a drug's efficacy.

The articles by Joost van de Heijden et al examined one aspect of this complex web of effects. Multidrug resistance (MDR) is a concept often considered in oncology. ${ }^{1}$ Multidrug resistant cells are thought to be resistant secondary to one or both of the following features: (a) lower intracellular drug concentrations via the pumping or extruding of drug (possibly in conjunction with compartmentalisation of the drug away from the site of action (for example, the nucleus)) and/or (b) altered susceptibility to the drug and increased repair mechanisms. ${ }^{2}$ Lower intracellular concentrations of the drug can occur by decreasing the uptake of the drug or enhancing its efflux. MDR is thought to occur through a family of "drug pumps" which extrude molecules from the cell. These "drug efflux pumps" belong to a superfamily of ATP dependent binding cassette transporters ( $\mathrm{ABC})$, including:
P-glycoprotein (Pgp, ABCB1), which affects lipophilic drugs preferentially; MDR associated proteins (MRPl-6, ABCCl-6), which affect anionic drugs preferentially; and breast cancer resistance protein (BCRP, ABCG2), which is associated with amphophilic drugs. ${ }^{3}$ Another mechanism for ridding the cell of a drug may be through distributing the drug into an acidic endosome and thereafter extruding the drug by an endosome mechanism. ${ }^{2}$ Thus, the concept of "drug resistance" in oncology is quite complex and there is no reason to expect that it will be less so in rheumatology.

\section{"The concept of drug resistance in RA as a consequence of 'drug efflux pumps' is attractive and is an important direction to follow"}

Joost van de Heijden et al, in their two papers, applied the concept of MDR to SSZ and, to some extent, to other DMARDs. They use human CEM $T$ lineage lymphocytic cells, an immortalised cancer $\mathrm{T}$ lymphocyte, in their studies. They demonstrated that SSZ resistance (defined as diminished antiproliferative effect of SSZ) occurs in this cell line and they also showed the kinetics of this acquired resistance. It takes four to six months to reach the resistance they deemed maximum at 1.5 $\mathrm{mM}$ SSZ, although they also point out that further resistance could be developed with more time (using $2.5 \mathrm{mM}$ SSZ). Resistance seems to disappear over a six month period but reappears within weeks of re-exposure to SSZ thereafter. The SSZ resistance correlates with the induction of the ABCG2 gene (also called the BCRP gene) and, coincidentally, with down regulation of ABCCl (also called MRPl). Thus, SSZ resistance was associated with induction of a drug efflux pump. A specific inhibitor of this pump decreased SSZ resistance by about 50\% (not $100 \%)$. This indicates that some but not all of the effects of SSZ resistance are due to this efflux pump. Although the authors could not explain the other
A somewhat complicating feature of their work is that the ABCCl (MRPl) is down regulated when ABCG2 is up regulated and is up regulated (and even overshoots baseline activity) when ABCG2 is down regulated towards baseline. One could imagine that the result of this inverse activity might easily affect other drugs given at the same time which are "pumped" by ABCCl. Interestingly, when cells became resistant to SSZ, they also had some increase in resistance to leflunomide and methotrexate. On the other hand, there was increased sensitivity to chloroquine, cyclosporin, and dexamethasone.

These data are fascinating as they may explain the resistance which occurs to DMARDs and, even, supply a rational approach to the use of some of these drugs together. Do these articles, in fact, explain drug resistance and can they be used for enhancing rational therapeutics in the rheumatic diseases?

Although these articles are, generally, quite well and carefully done, some notes of caution are needed. Most importantly, the cell type that is being tested is not a rheumatoid arthritis cell. The authors comment on this, but the problem remains. It appears, for example, that different cell types have different sensitivities. In an article by Bergman et al, dexamethasone, but not cortisol, decreased sensitivity to an oncological drug in a non-small lung cancer cell, unless verapamil was used, when both cortisol and dexamethasone produced resistance to the oncological drug. ${ }^{5}$ Thus, one needs to be cautious about applying the results using CEM T cells directly to rheumatoid arthritis. Further, the active component of SSZ in rheumatoid arthritis, sulfapyridine, was not active in this cell system and conferred no resistance to CEM T cells, according to the authors. Again, it is hard to understand how the SSZ resistance can apply in rheumatoid arthritis without an effect by sulfapyridine. Finally, it would be important to see if this also applies ex vivo, an admittedly difficult proposition.

Despite these issues, the concept of drug resistance in rheumatoid arthritis as a consequence of induction of "drug efflux pumps" is attractive and is an important direction to follow. Understanding the appearance of drug resistance and applying the MDR gene concept to rheumatology may eventually improve our ability to treat patients effectively and rationally.

Ann Rheum Dis 2004;63:115-116.

doi: 10.1136/ard.2003.014613 
Correspondence to: Dr D E Furst, UCLA Medical School, 1000 Veteran Avenue, Rehabilitation Center, Room 32-59, Los Angeles, USA; defurst@mednet.ucla.edu

\section{REFERENCES}

1 Bradshaw DM, Arceci RJ. Clinical relevance of drug efflux as a mechanism of multidrug resistance. J Clin Oncol 1998;16:3674-90.
2 Raghunand N, Martinez-Zaguilan, Wright SH, Gillies RJ. PH and drug-resistance. II Turnover of acidic vesicles and resistance to weekly basic chemotherapeutic drugs. Biochem Pharmacol 1999;57:1047-58.

3 van der Heijden J, de Jong MC, Dijkmans BAC, Lems WF, Oerlemans R, Kathmann I, et al. Acquired resistance of human $T$ cells to sulfasalazine: stability of the resistant phenotype and sensitivity to non-related DMARDs. Ann Rheum Dis 2004:63:131-7.
4 van der Heijden J, de Jong MC, Dijkmans BAC, Lems WF, Oerlemans R, Kathmann I, et al. Development of sulfasalazine resistance in human $T$ cells induces expression of the multidrug resistance transporter $A B C G 2$ (BCRP) and augmented production of TNF $\alpha$. Ann Rheum Dis 2004;63:138-43.

5 Bergman AM, Pinedo HM, Peters GJ. Steroids affect collateral sensitivity to gecitabine of multidrug resistant human lung cancer cells. Eur J Pharmacol 2001;416:19-24.

\section{Get published within days of acceptance with \\ ARD}

We are delighted to announce that the Annals of the Rheumatic Diseases is launching a "publish ahead of print" programme from February 2004. Selected papers will be fast tracked and published online months before they appear in the print journal.

Papers of major significance to the international rheumatology community will be published within days of acceptance. The published article will be the raw accepted manuscript; edited and typeset versions will also be published as soon as they are available.

In addition to being available on ARD Online, the publish ahead of print articles will be searchable through PubMed/Medline-establishing primacy for your work. They will be linked from the ARD Online home page.

$A R D^{\prime} s$ publish ahead of print programme is unique among the major rheumatology journals - to take advantage of this service submit your papers to Annals of the Rheumatic Diseases using our online submission and review system Bench>Press (http://submit-ard. bmijournals.com). For further information contact ARD@bmigroup.com. 